\title{
Sexual health clinics for women led by specialist nurses or senior house officers in a central London GUM service: a randomised controlled trial
}

\author{
K Miles, N Penny, D Mercey, R Power
}

Sex Transm Infect 2002; 78:93-97

See end of article for authors' affiliations

Correspondence to: Kevin Miles, Department of Sexually Transmitted Diseases, Mortimer Market Centre, off Capper Street, London WCIE 6AU, UK; kmiles@gum.ucl.ac.uk

Accepted for publication 13 December 2001

\begin{abstract}
Objectives: To assess the care process and clinical outcomes for two different models of GUM clinic for women: one led by specialist nurses and the other by senior house officers (SHOs)

Method: An open randomised controlled trial was carried out in a central London genitourinary medicine (GUM) women's clinic. Of 1172 women telephoning for an appointment, 880 were randomised to provide 169 eligible patients in the specialist nurse arm and 178 in the SHO arm. Of the eligible patients a total of 224 attended their appointment. The clinical records of the randomised women were audited for adequacy of care according to local guidelines. 30 key variables were objectively assessed and recorded on a standard audit form. An overall unitary index score (\%) was calculated for each patient. The main variables associated with the outcome of specialist nurse and $\mathrm{SHO}$ decision making (diagnostic test request, preliminary diagnosis, and treatment provided) were then analysed independently.

Results: The median documentation audit scores for specialist nurses $(n=103)$ and SHOs $(n=121)$ were $92 \%$ and $85 \%$ respectively $(p<0.0001)$. The specialist nurses' documentation was significantly $(p<0.05)$ more complete than the SHOs' for five variables: details of menstrual cycle, physical examination, medication instructions given to patients, health promotion discussion, and provision of condoms. Specialist nurses performed equally to the SHOs with regard to requesting the correct diagnostic tests, providing the correct preliminary diagnosis, and providing the correct treatment.

Conclusions: A model of care using trained GUM nurses working within agreed protocols can provide comprehensive patient care for female patients that is equal to care provided by SHOs. Our results raise important issues regarding advanced GUM nursing education and training, protocol development, and accountability.
\end{abstract}

n 1988 a Department of Health report ${ }^{1}$ recommended that the roles of nurses and doctors in genitourinary medicine (GUM) clinics be examined, a contention reinforced by research conducted in $1992 .^{2}$ The latter study recommended that serious consideration be given to the introduction (or extension) of nurse run clinics.

More recently, the government signalled its commitment to strengthen the nursing contribution to health and health care. ${ }^{3}$ A major reconstruction of medical and nursing roles has also been suggested in order to involve the skills of all professionals in solving health problems. ${ }^{45}$

In January 1999, a new model of care was initiated at this GUM service whereby experienced, specialist GUM nurses were trained to coordinate the first line, comprehensive care of female patients. "Comprehensive care nurse led clinics" were implemented primarily as a nursing development opportunity to complement the existing "doctor led" clinics. The key difference between the two models of care was that the doctors coordinated care for individual women with task delegated input from a team of staff nurses, whereas the specialist nurses coordinated a "one stop shop" model in which the majority of the care was delivered by one person.

Throughout the implementation of comprehensive care nurse led clinics, a process evaluation, including staff interviews and cost analysis, assessed the extent to which the nurse led initiative was implemented as intended. Six months after the introduction of comprehensive care nurse led clinics, a randomised controlled trial (RCT) was conducted to assess whether the clinical outcomes of clinics led by specialist nurses were at least as good as those of clinics led by senior house officers (SHOs). A patient satisfaction survey was also developed and conducted during this same period although the survey respondents did not originate from the randomised sample. These results are not presented here.

This paper reports on the care processes and clinical outcomes of the randomised controlled trial aspect of the nurse led clinic evaluation.

\section{METHODS}

Experience and training of specialist nurses and SHOs

In January 1999, two F grade specialist nurses, each with over 3 years' GUM nursing experience, were appointed to conduct comprehensive care nurse led sexual health clinics. They had a 2 week induction and training period followed by regular clinical supervision with a consultant physician. The specialist nurses' defined role entitled them to work according to local protocols, to coordinate the comprehensive care of women who were undifferentiated from those seen by doctors. They worked autonomously, without necessarily asking the advice of a doctor. They were able to elicit a sexual history, perform a genital examination, collect specimens, and provide the preliminary diagnosis, results, selected treatments, and health promotion for women presenting with various sexual health conditions and issues. They were required to work within the boundaries of their own knowledge and competence, and refer cases beyond their clinical expertise (approximately 12\% of their workload) to the medical staff.

The nurse led clinics ran alongside the traditional model of GUM care involving a doctor leading patient care supported by a team of staff nurses. In this model of care the doctor elicits the sexual history, and depending on the presenting problem 


\section{Key messages}

- Innovative models of STI management utilising the skill and expertise of all members of the multidisciplinary team need to be developed for the future of GUM

- This is the first known randomised controlled trial of its kind in the GUM setting

- Given the appropriate training and ongoing support, a model of care using experienced GUM nurses can provide safe, acceptable, and effective care

and previous attendance history, performs the physical examination and collects the relevant specimens, or delegates these tasks to the staff nurses. The doctor then provides the preliminary diagnosis, results, and treatments. The sexual health promotion aspects of care are dealt with by input from both the doctor and the staff nurses.

SHOs rotate through the service for a 6 month training period. The SHOs involved in the study commenced their GUM training rotation in February 1999. Following a l week induction period, the SHOs commenced doctor led clinics as described above. The SHOs had a weekly group clinical supervision session in addition to individual supervision with a consultant physician. At the time of the outcome evaluation, the SHOs had more than 4 months' GUM experience and covered approximately $35 \%$ of the female clinic workload. Similar to the specialist nurses, the SHOs had direct access to senior medical staff for referring cases beyond their clinical experience.

\section{Study design and sample size}

The study was conducted in the women's clinic of a central London genitourinary medicine (GUM) clinic with approximately 22000 female patient attendances/year. The study used an open randomised controlled trial design.

In June 1999, for a period of 9 weeks, female patients telephoning for an appointment were randomised to see either a doctor or specialist nurse. From the randomised patients, we selected only those who saw a SHO for the analysis. SHOs were chosen as controls as this was the minimum level of care expected of the specialist nurses. We aimed to show that specialist nurses were not inferior to SHOs (that is, a score no more than five points less than SHOs on average) with respect to documenting the sexual history, requesting tests, documenting the examination, providing the correct preliminary diagnosis and treatment, and documenting any sexual health promotion. A sample size calculation indicated 130 would be required in each arm, providing $90 \%$ power assuming nurses truly scored no more than three points less than SHOs on average, using two tailed tests.

\section{Sampling strategy}

A computer program was used to generate three simple random sequences in order to allocate patients to specialist nurse:doctor in ratios of 1:3, 1:4, and 1:5 with block sizes of eight, 10, and 12. The different ratios were generated to account for the daily changes in appointment availability. For example, if there were not enough specialist nurse appointments available, the ratio could be changed to account for this. The telephone reception staff were provided with a simple table listing the randomisation allocations, with a space next to each allocation to enter the patient clinic reference number as the appointment was booked. The reception staff were not blinded to the randomisation allocations although to reduce any selection bias, the time of appointment booking was recorded next to each allocation and regular supervision of the reception staff performing the randomisation ensured that no favouring of group allocation occurred. Reception staff do not perform any triage of patients and therefore it was not in their interests to influence the order of enrolment.
Not all patients seeing specialist nurses and SHOs during the study time were randomised. Some patients attended the service without an appointment and there were times when the telephone reception staff were too busy to carry out the randomisation. However, all staff at clinic level were blinded to which patients had been randomised.

Patients did not consent at the time of randomisation. All patients were informed of the study when they presented at the clinic and were given an opportunity to opt out of the randomisation if unhappy with their allocated practitioner. Post-randomisation pre-intervention consent served as a solution to the issues of consenting patients over the telephone.

The local research ethics committee approved the study.

\section{Outcome measurement}

The clinical records of the randomised women were audited for adequacy of care according to local guidelines. An audit proforma listed 30 key variables in addition to a section to record the patient's date of birth, ethnic origin, and presenting condition. The 30 variables reflected details listed in the patient case note proforma used to document patient management during all doctor and nurse led consultations. Objective criteria were established on how to judge each variable before allocating a score between 0 and 1 . For example, a score of 0 was given if details of the sexual history were not recorded, 0.5 if there were incomplete data, and a score of 1 if a complete sexual history was recorded. In instances when no information was required-for example, when the patient did not require an examination, a non-applicable option was available. A unitary index score was calculated for each set of patient case notes and then converted to a percentage for analysis. The main variables associated with the outcome of specialist nurse and SHO decision making (diagnostic test request, preliminary diagnosis, and treatment provided) were then analysed independently.

Since we were essentially comparing two different models of care, documentation by staff nurses supporting the SHOs and documentation from doctors supporting the specialist nurses was also counted. Cases that were difficult to interpret (15/224) were discussed with a consultant physician (DM) and a consensus reached.

\section{Reliability}

The reviewer (KM) was not blinded to the identity of the practitioners. However, 50\% (112/224) of clinical records were randomly selected and reviewed for score reliability by a second person (DM). Pearson's correlation coefficient was used to measure the strength of the association between the final unitary index scores of the reviewers. For each of the 30 items used to create the unitary index score, the proportion of cases where the two reviewers agreed as to whether the item was recorded or not was then computed to determine those items where discrepancy between reviewers was greatest.

\section{Analysis}

Data from the audit forms were single entered and analysed using sPss. An intention to treat analysis was chosen-that is, if a patient was randomised to see a specialist nurse but was referred to a senior doctor in the course of the appointment, the data were analysed within the specialist nurse arm. The $\chi^{2}$ test, and Fisher's exact test where appropriate, were used to compare the specialist nurse and SHO groups with respect to rates of attendance and the individual variables. Nonparametric Mann-Whitney $U$ tests were used to compare the unitary index scores between specialist nurse and SHO groups.

\section{RESULTS}

Figure 1 shows the flow of patients through the randomisation; 1172 patients telephoned for an appointment; 880 of 


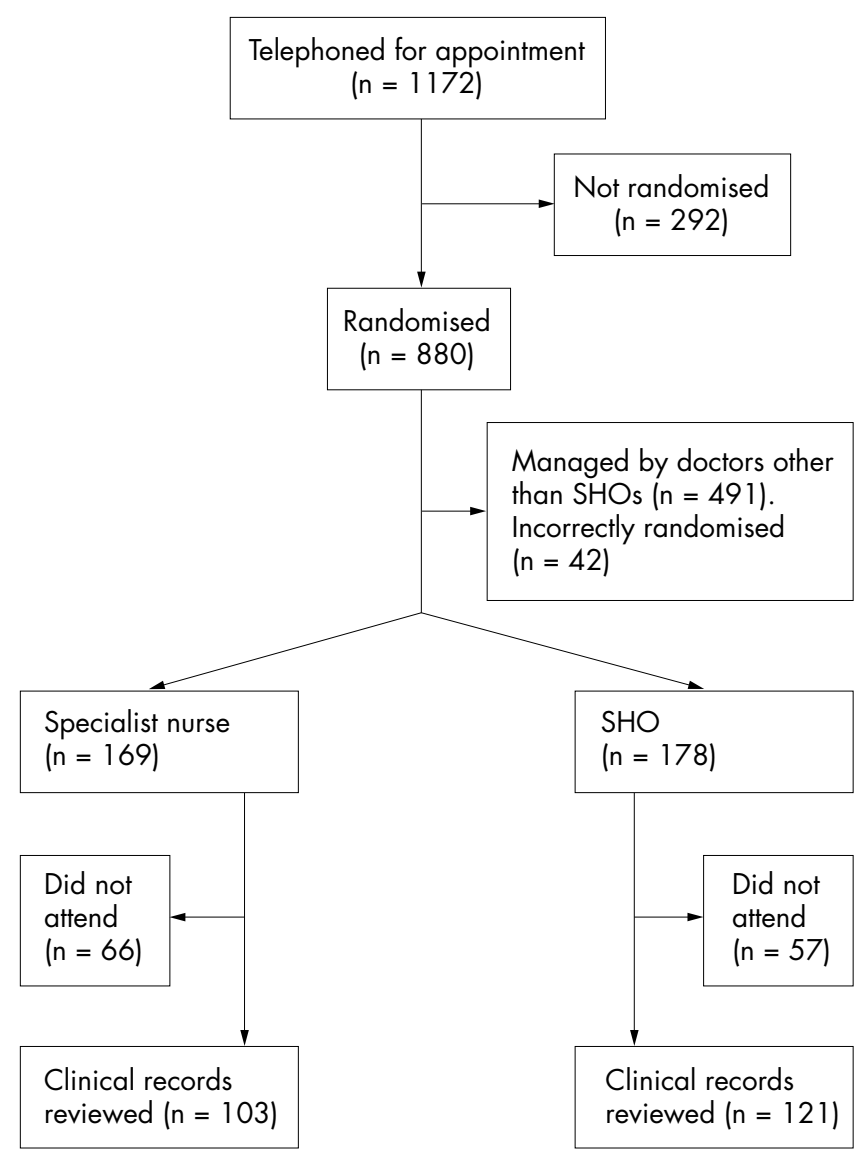

Figure 1 Randomisation flow chart.

these were randomised to provide 169 patients in the specialist nurse arm and 178 in the SHO arm, of whom 103 and 121 respectively kept their appointment. There was no statistically significant difference in non-attendance rates between specialist nurse and SHO groups (specialist nurse 66/169 (39\%) $v$ SHO 57/178 (32\%) $p=0.2)$. The unusually high nonattendance rate seen in the sample population reflected the June 1999 attendance figures for the female clinic that peaked at $33.3 \%$ non-attendance for all new patient appointments. The remaining 533 patients allocated to doctors other than SHOs $(n=491)$, or incorrectly randomised by reception staff $(\mathrm{n}=42)$, were not included in the analysis as the comparison was with SHOs only. Two hundred and ninety two women were not randomised, the main reason being that these women made specific requests for particular dates, times, or practitioner. Apart from non-attenders, no patient opted out following randomisation.

All clinical records from 103 specialist nurse and 121 SHO clinic attendees were audited. The two groups of patients were comparable in terms of age, ethnic origin, and presenting problem (table 1). The median unitary index scores for specialist nurse and SHO groups were 92\% (range 32-100) and $85.2 \%$ (range $20-100)$ respectively $(\mathrm{p}<0.0001)$. There was no significant difference between SHO and specialist nurse documentation for 25 of the 30 variables assessed. The five variables in which the specialist nurses' documentation was significantly $(p<0.05)$ more complete than the SHOs' included details of menstrual cycle, physical examination, medication instructions given to patients, health promotion discussion, and provision of condoms.

Specialist nurses performed equally to the SHOs with regard to requesting the correct diagnostic tests (table 2), providing the correct preliminary diagnosis (table 3), and providing the correct treatment (table 4). The two cases in which a specialist nurse and two cases in which a SHO supplied treatment outside the local clinic guidelines are reported in table 5.
Table 1 Characteristics of patients and presenting conditions

\begin{tabular}{|c|c|c|}
\hline & $\begin{array}{l}\text { Specialist nurse } \\
\text { group }(n=103)\end{array}$ & $\begin{array}{l}\text { SHO group } \\
(n=121)\end{array}$ \\
\hline Median (range) age (years) & $28.6(18-53)$ & $28.7(17-52)$ \\
\hline \multicolumn{3}{|l|}{ Ethnic origin } \\
\hline White & $72(70)$ & $85(70)$ \\
\hline Black African & $6(6)$ & $5(4)$ \\
\hline Black Caribbean & 3 (3) & $3(2)$ \\
\hline Black other & $1(1)$ & $3(2)$ \\
\hline Indian & $1(1)$ & $2(2)$ \\
\hline Chinese & $4(4)$ & $1(1)$ \\
\hline Asian other & $3(3)$ & $2(2)$ \\
\hline Other ethnic group & $3(3)$ & 1 (1) \\
\hline Missing data & $10(10)$ & $19(16)$ \\
\hline \multicolumn{3}{|l|}{ Presenting condition } \\
\hline $\begin{array}{l}\text { Asymptomatic blood test } \\
\text { and/or check up }\end{array}$ & $26(25)$ & $33(27)$ \\
\hline $\begin{array}{l}\text { Symptomatic vaginal } \\
\text { discharge/dysuria/irritation }\end{array}$ & $42(41)$ & $39(32)$ \\
\hline Genital ulcer/warts & $7(7)$ & $10(8)$ \\
\hline Pelvic pain & $4(4)$ & $8(7)$ \\
\hline Results & $9(9)$ & $16(13)$ \\
\hline Other & $15(15)$ & 15 (12) \\
\hline
\end{tabular}

Table 2 Diagnostic test requests* by specialist nurses and $\mathrm{SHOs}$

\begin{tabular}{lll}
\hline & Specialist nurse $(\mathrm{n}=92)$ & SHO $(\mathrm{n}=99)$ \\
\hline Correct & $89(96.7)$ & $89(90.8)$ \\
Too few tests requested & $3(3.3)$ & $9(9.2)$ \\
\hline
\end{tabular}

$\mathrm{p}=0.09$. Data are number $(\%) ;{ }^{*}$ not all patients required diagnostic tests to be performed.

Table 3 Preliminary diagnosis* recorded by specialist nurses and $\mathrm{SHO}$

\begin{tabular}{lll}
\hline & $\begin{array}{l}\text { Specialist } \\
\text { nurse }(\mathbf{n}=92)\end{array}$ & $\begin{array}{l}\text { SHO } \\
(\mathbf{n}=103)\end{array}$ \\
\hline Correct & $76(82.6)$ & $80(77.7)$ \\
Not recorded & $12(13.0)$ & $16(15.5)$ \\
Correct but doesn't fit with clinic guidelines & $4(4.3)$ & $7(6.8)$ \\
\hline
\end{tabular}

$p=0.6$. Data are number $(\%) ;{ }^{*}$ not all patients required a preliminary diagnosis to be made.

Table 4 Treatment* recorded by specialist nurses and $\mathrm{SHOs}$

\begin{tabular}{lll}
\hline & $\begin{array}{l}\text { Specialist nurse } \\
(n=49)\end{array}$ & $\begin{array}{l}\text { SHO } \\
(n=59)\end{array}$ \\
\hline $\begin{array}{l}\text { Correct } \\
\text { Incorrect according to clinic guide }\end{array}$ & $\begin{array}{l}47(95.9) \\
\text { (4.1) }\end{array}$ & $\begin{array}{l}57(96.6) \\
2(3.4)\end{array}$ \\
\hline$p=0.6$. Data are number (\%); *not all patients required treatment.
\end{tabular}

However, it must be noted that at the time of the study, patient group directions were not being used and, therefore, specialist nurses were required to have all treatment decisions confirmed by a doctor.

The review of clinical records by a second person found the scoring system to be reliable. A Pearson's correlation coefficient of $0.886(\mathrm{p}<0.001)$ indicated a strong association between the final unitary index scores of the two reviewers. There was a high proportion of cases in which score agreement was reached for each of the 30 individual items 
Table 5 Treatments provided outside clinic guidelines by specialist nurses ( $\mathrm{SpN}$ ) and $\mathrm{SHO}$

SpN93 Clotrimazole pessaries given for trichomoniasis. Patient breast feeding

SpN78 Clindamycin gel given for vaginal discharge of unknown origin

SHO127 Eight clotrimazole pessaries given at patient's request without any confirmed candidiasis diagnoses in over 2 years

SHO135 Metronidazole immediately followed by another dose in 72 hours

\begin{tabular}{|c|c|c|c|}
\hline & $\begin{array}{l}\text { Specialist nurse } \\
(\mathrm{n}=60)\end{array}$ & $\begin{array}{l}\mathrm{SHO} \\
(\mathrm{n}=52)\end{array}$ & p Value \\
\hline Reviewer 1 & 90.3 & 83.9 & 0.008 \\
\hline Reviewer 2 & 92.6 & 85.7 & 0.002 \\
\hline
\end{tabular}

(median 98; range 78-100). The areas in which there was a difference in scores were those that required more subjective measurement. For example, there was only $88 \%$ scoring agreement in the item documenting the physical examination (data not shown). Although criteria were set on how to measure each item, there was still a difference in what each reviewer perceived to be complete documentation. However, it is important to note that the unitary index scores of both reviewers consistently showed specialist nurses' documentation to be more complete than SHOs' (table 6).

\section{DISCUSSION}

Although "extended roles" of GUM nurses have been evident for some time, ${ }^{6}$ formalised comprehensive care nurse led sexual health clinics are still a relatively new concept in the United Kingdom. Recent accounts of change to UK GUM nursing practice have been reported, ${ }^{78}$ but no rigorous studies against which to judge our results have been performed. However, studies in other fields of health care have shown that nurses are capable of carrying out procedures usually the domain of doctors, often to the greater satisfaction of patients. $^{9-13}$

Our results show that trained, experienced specialist nurses supported within the multidisciplinary team are at least as effective in the assessment and management of female patients as SHOs. There were no serious clinical errors made by the specialist nurses, and the documentation of the process of care was as good, and in some respects more complete, than SHOs. The specialist nurses scored significantly more highly in the documentation of the physical examination, medication instructions given to patients, health promotion, and provision of condoms.

There are a number of plausible explanations for these results. One could suggest that nurses in general, are better at documenting patient care, or that the specialist nurses documented more conscientiously while their new role was under scrutiny. An alternative explanation is that the "one stop shop" model of care provided the specialist nurses with more time to document patient care.

One limitation of the study is that the SHOs had limited experience of working in a GUM environment, but this situation is true of all SHOs who rotate through the service on a 6 monthly basis. Although relatively inexperienced, these SHOs were generally supported by a team of experienced staff nurses who contributed to the documentation of care whereas the specialist nurses were mostly working alone, only accessing advice for cases beyond their clinical expertise and for medication prescription. Therefore, in practice, neither the SHOs nor specialist nurses truly worked independently. The specialist nurses needed to access a doctor for a prescription in
$48 \%(49 / 103)$ of cases. We were unable to determine from the clinical records how many of the treatment decisions made by the SHOs also required input from senior doctors and other colleagues within the multidisciplinary team. As a result, the differences in our outcomes cannot be attributed to the profession of the practitioner alone, but as a possible result of the different models of care within which the practitioners worked.

Several methodological weaknesses of the study are also apparent. Firstly, we did not blind the reception staff to the randomisation allocations. The practicalities of managing and motivating staff to use a masked system (for example, a sealed envelope approach) may have led to poor enrolment in addition to service disruption. None the less, our randomisation procedures led to the two groups of patients being comparable in terms of age, ethnic origin, and presenting problem (table 1).

Secondly, resources were not available to transcribe all 224 patient clinical records to create masked conditions. With a nurse researcher (KM) conducting the audit of clinical records under non-masked conditions, measurement bias may have been introduced to the study. However, steps were taken at the outset to reduce any such effects. Clear, objective criteria were created for the audit and the review of $50 \%$ of all clinical records by a second person showed inter-reviewer score reliability.

The burden of sexually transmitted infections in England is substantial and shows no sign of waning. ${ }^{14}$ Team working across professional boundaries, flexible working to make the best use of the range of skills and knowledge which staff have, and developing new, more flexible career pathways for staff are key areas of emphasis for progressing health services to meet the demands of the public. ${ }^{15}{ }^{16}$ The National Strategy for Sexual Health and HIV builds on this by proposing that nurses will have an expanding role in the management of sexually transmitted infections as specialists and consultants. ${ }^{17}$

The benefits of nurses providing first line sexual health care are many. The skills of experienced nurses can be fully realised and these nurses therefore retained in the specialty, and doctors relocated from routine services can concentrate on more medically complex aspects of service provision. However, if nurses are to take on more advanced roles, local protocols must be in place to ensure and enhance professional practice and accountability. Finally, education agendas need reviewing in light of the advanced skills required by such nurses. Educationalists need to respond to the new skills and knowledge that nurses need in not only providing direct patient care, but in the development of practice protocols, patient group directions, and clinical governance initiatives to monitor and maintain standards of patient care.

\section{CONTRIBUTORS}

NP and DM initiated the development of nurse led clinics; all authors contributed to the design of the study; KM supervised the randomisation, collected and analysed the data with support from RP and DM; KM prepared the preliminary draft of the manuscript with contributions from all authors.

\section{ACKNOWLEDGEMENTS}

Data from this paper were presented as an oral presentation (abstract No 019) at the Joint ASTDA/MSSVD STIs at the Millennium conference, Baltimore, USA, 3-6 May 2000. 
KM holds a nurse research fellowship funded by Camden and Islington Community Health Services NHS Trust.

We thank the specialist nurses Heather MacDougal and Liz Rees who have contributed to the development and study of nurse led clinics; the doctors, nurses, reception staff, and patients of Mortimer Market Centre who contributed to and participated in the study; Andrew Copas for statistical advice; Professor Ian Weller, Professor Anne Johnson, and Dr Angela Robinson for reviewing drafts of this paper.

Conflicts of interest: none.

\section{Authors' affiliations}

K Miles, D Mercey, R Power, Department of Sexually Transmitted Diseases, Royal Free and University College Medical School, University College London, Mortimer Market Centre, London, UK

N Penny, Camden and Islington Community Health Services NHS Trust, Mortimer Market Centre, London, UK

\section{REFERENCES}

1 Department of Health. Report of the working group to examine workloads in genito-urinary medicine clinics. London: DoH, 1988.

2 Allen I, Hogg D. Work roles and responsibilities in genitourinary medicine clinics. London: Policy Studies Institute, 1993.

3 Department of Health. Making a difference. Strengthening the nursing, midwifery and health visiting contribution to health and healthcare. London: DoH, 1999.

4 Allen I. Modernising the NHS: challenges to the health services: the professions. BM 2000;320:1533-5
5 Salvage J, Smith R. Doctors and nurses: doing it differently. BM 2000;320:1019-20.

6 Rogers JS, Adler MW. Role and training of nurses working in departments of genitourinary medicine in England and Wales. 1. Role. Genitourin Med 1987:63:122-6.

7 Allen D. Putting the experts in charge. Nurs Stand 1998;12:22-3.

8 Sutton A, Kingsland T, Ross S, et al. Nursing in genitourinary medicine: 10 years on from the Monks Report. Int J STD AIDS 1999; 10:539-42.

9 Campbell NC, Thain J, Deans HG, et al. Secondary prevention clinics for coronary heart disease: randomised trial of effect on health. BM 1998;316:1434-7.

10 Hill J, Bird HA, Harmer R, et al. An evaluation of the effectiveness, safety and acceptability of a nurse practitioner in a rheumatology outpatient clinic. BrJ Rheumatol 1994;33:283-8.

11 Kinnersley $\mathbf{P}$, Anderson E, Parry K, et al. Randomised controlled trial of nurse practitioner versus general practitioner care for patients requesting "same day" consultations in primary care. BM 2000;320:1043-8.

12 Shum C, Humphreys A, Wheeler D, et al. Nurse management of patients with minor illnesses in general practice: multicentre, randomised controlled trial. BM 2000;320:1038-43.

13 Venning $\mathbf{P}$, Durie $A$, Roland $M$, et al. Randomised controlled trial comparing cost effectiveness of general practitioners and nurse practitioners in primary care. BM 2000;320:1048-53.

14 Lamagni TL, Hughes G, Rogers PA, et al. New cases seen at genitourinary medicine clinics: England 1998. Commun Dis Rep 1999:9:S1-12.

15 Department of Health. A health service of all the talents: developing the NHS workforce-consultation document on the review of workforce planning. London: DoH, 2000.

16 Department of Health. The NHS Plan-a plan for investment. A plan for reform. London: DoH, 2000

17 Department of Health. The national strategy for sexual health and HIV. London: DoH, 2001.

For just \$8 you can purchase the full text of individual articles using our secure online ordering service. You will have access to the full text of the relevant article for 48 hours during which time you may download and print the pdf file for personal use.

www.sextransinf.com 